BULLETIN OF THE POLISH

ACADEMY OF SCIENCES

MATHEMATICS

Vol. 68, No. 1, 2020

\title{
On barycenters of probability measures \\ by
}

\author{
Sergey BEREZIN and Azat MIFTAKHOV
}

Presented by Rafał LATAEA

Summary. A characterization is presented of barycenters of the Radon probability measures supported on a closed convex subset of a given space. A case of particular interest is studied, where the underlying space is itself the space of finite signed Radon measures on a metric compact and where the corresponding support is the convex set of probability measures. For locally compact spaces, a simple characterization is obtained in terms of the relative interior.

1. The main goal of the present note is to characterize the barycenters of the Radon probability measures supported on a closed convex set. Let $X$ be a Fréchet space. Without loss of generality, the topology on $X$ is generated by the translation-invariant metric $\rho$ on $X$ (for details see [2]).

We denote the set of Radon probability measures on $X$ by $\mathcal{P}(X)$. The barycenter $a \in X$ of a measure $\mu \in \mathcal{P}(X)$ is, by definition,

$$
a=\int_{X} x \mu(d x),
$$

if the latter integral exists in the weak sense, that is,

$$
\Lambda a=\int_{X} \Lambda x \mu(d x)
$$

for all $\Lambda \in X^{*}$, where $X^{*}$ is the topological dual of $X$. More details on such integrals can be found in [2, Chapter 3].

2020 Mathematics Subject Classification: Primary 28C05, 46N30, 60B05, 60B11.

Key words and phrases: barycenters of measures, characterization of barycenters, space of Radon measures.

Received 28 November 2019; revised 3 March 2020.

Published online 20 April 2020. 
Note that if (1) exists, then

$$
a=\int_{\operatorname{supp} \mu} x \mu(d x)
$$

and, by the Hahn-Banach separation theorem, $a \in \overline{\operatorname{co}(\operatorname{supp} \mu)}$, where $\operatorname{co}(\cdot)$ stands for the convex hull. From now on, we will use the bar over a set to denote its topological closure.

The following theorem gives a characterization of the barycenters of measures from $\mathcal{P}(X)$.

TheOREM 1. Let $M \subset X$ be a non-empty compact convex set, and let $a \in M$. Then the following statements are equivalent:

(i) There exists $\mu \in \mathcal{P}(X)$ with $\operatorname{supp} \mu=M$ and with barycenter a.

(ii) We have

$$
M=\overline{V_{a}},
$$

where $V_{a}=\{x \in M \mid \exists \alpha>0:-\alpha x+(1+\alpha) a \in M\}$.

REMARK 1. We note that the condition (4) is non-local and concerns the whole set $M$.

REMARK 2. We require $M$ to be compact in order to ensure the separability of $M$ and the existence of weak integrals (see, e.g., [2, Theorem 3.27]). If $X$ is finite-dimensional, the theorem holds without this requirement.

Proof of Theorem 1. (a) First, we prove that (i) $\Rightarrow$ (ii). Let $c \in M$ and let $U_{\delta}(c)$ be the open ball of radius $\delta>0$ centered at $c$. Because $M$ is the support of $\mu$, one has $\mu\left(U_{\delta}(c)\right)>0$. Also, since $M$ is compact, so is $\overline{U_{\delta}(c) \cap M}$, and

$$
c_{\delta}=\frac{1}{\mu\left(U_{\delta}(c)\right)} \int_{U_{\delta}(c)} x \mu(d x) \in M
$$

is well-defined. It is easy to show that

$$
\lim _{\delta \rightarrow+0} c_{\delta}=c
$$

in the weak topology $\sigma\left(X, X^{*}\right)$. Indeed, take any $\Lambda \in X^{*}$. Since $\Lambda$ is continuous, for every $\varepsilon>0$ there exists $\delta_{0}>0$ such that $x \in U_{\delta_{0}}(c)$ implies $|\Lambda(x)-\Lambda(c)|=|\Lambda(x-c)|<\varepsilon$. Then, it follows from the definition of the weak integral that

$$
\left|\Lambda\left(c_{\delta}-c\right)\right| \leq \frac{1}{\mu\left(U_{\delta}(c)\right)} \int_{U_{\delta}(c)}|\Lambda(x-c)| \mu(d x)<\varepsilon
$$

whenever $\delta \in\left(0, \delta_{0}\right)$. This means that $c_{\delta} \rightarrow c$ in the weak topology as $\delta \rightarrow+0$. 
Further, for any $\delta>0$, either $\mu\left(U_{\delta}(c)\right)=1$ or $0<\mu\left(U_{\delta}(c)\right)<1$. We show that in both cases $c_{\delta} \in V_{a}$. Indeed, if $\mu\left(U_{\delta}(c)\right)=1$, then $c_{\delta}=a$ and thus $c_{\delta} \in V_{a}$. If $0<\mu\left(U_{\delta}(c)\right)<1$, set

$$
\tilde{c}_{\delta}=\frac{1}{\mu\left(X \backslash U_{\delta}(c)\right)} \int_{X \backslash U_{\delta}(c)} x \mu(d x) \in M .
$$

Clearly, $\alpha c_{\delta}+(1-\alpha) \tilde{c}_{\delta} \in M, \alpha \in[0,1]$, by convexity. Moreover, $a=$ $\mu\left(U_{\delta}(c)\right) c_{\delta}+\left(1-\mu\left(U_{\delta}(c)\right)\right) \tilde{c}_{\delta}$. Therefore, by a simple geometric argument and by the definition of $V_{a}$, it is clear that $c_{\delta} \in V_{a}$.

Since $X$ is a locally convex space and since $V_{a}$ is convex, the closures of $V_{a}$ in the weak and original topologies coincide. Consequently, by passing to the limit $\delta \rightarrow+0$, one arrives at

$$
c=\lim _{\delta \rightarrow+0} c_{\delta} \in \overline{V_{a}} .
$$

This concludes the proof of the claim.

(b) We prove that (ii) $\Rightarrow(\mathrm{i})$ by constructing $\mu \in \mathcal{P}(X)$ with support $M$ and barycenter $a$.

Being a metric compact, $M$ is separable, hence there exists $M_{0}$ such that $\overline{M_{0}}=M=\overline{V_{a}}$. Without loss of generality, one can think that $M_{0}=$ $\left\{x_{n}\right\}_{n=1}^{\infty} \subset V_{a}$ and $\overline{\left\{x_{n}\right\}_{n=1}^{\infty}}=M$. By the definition of $V_{a}$, there exist $\left\{\alpha_{n}\right\}_{n=1}^{\infty}$ such that $\alpha_{n}>0$ and $-\alpha_{n} x_{n}+\left(1+\alpha_{n}\right) a \in M$.

Let us define the discrete measure

$$
\mu=\sum_{k=1}^{\infty} \frac{1}{2^{n}} \cdot \frac{\alpha_{n} \delta_{x_{n}}+\delta_{-\alpha_{n} x_{n}+\left(1+\alpha_{n}\right) a}}{1+\alpha_{n}},
$$

where $\delta_{x}$ is the delta measure at $x$. Clearly, this is a Radon probability measure, and a simple computation shows that its barycenter is $a$. Indeed, for every $\Lambda \in X^{*}$ one has

$$
\int_{X} \Lambda x \mu(d x)=\sum_{k=1}^{\infty} \frac{1}{2^{n}} \cdot \frac{\alpha_{n} \Lambda x_{n}+\left(-\alpha_{n} \Lambda x_{n}+\left(1+\alpha_{n}\right) \Lambda a\right)}{1+\alpha_{n}}=\Lambda a .
$$

It remains to prove that $\operatorname{supp} \mu=M$. First, we note that $\left\{x_{n}\right\}_{n=1}^{\infty} \subset \operatorname{supp} \mu$. Consequently, $M=\overline{\left\{x_{n}\right\}_{n=1}^{\infty}} \subset \operatorname{supp} \mu$, and therefore $M \subset \operatorname{supp} \mu$. By the definition (10) one also has supp $\mu \subset M$, which concludes the proof.

Further, we will use the following standard notation from convex analysis. For $a, b \in X$ we define the (line) segment $[a, b]$ and the open (line) segment $(a, b)$ to be

$$
\begin{aligned}
& {[a, b]=\{x \in X \mid x=(1-\lambda) a+\lambda b, \lambda \in[0,1]\},} \\
& (a, b)=\{x \in X \mid x=(1-\lambda) a+\lambda b, \lambda \in(0,1)\} .
\end{aligned}
$$


Let us recall that the relative interior of a set $M$ is

$$
\operatorname{relint}(M)=\{x \in M \mid \exists U(x): U(x) \cap \operatorname{aff}(M) \subset M\},
$$

where $U(x)$ is an open neighborhood of $x$ and aff $(M)$ is the affine hull of $M$. Also, we recall that the relative algebraic interior of $M$ is the set

$$
\operatorname{core}(M)=\{x \in M \mid \forall y \in \operatorname{aff}(M) \exists \alpha>0:[x,-\alpha y+(1+\alpha) x] \subset M\} .
$$

It is well-known that any locally compact topological vector space is finite-dimensional (see, e.g., [2]), in which case the following corollary holds.

Corollary 1.1. If $X$ is a locally compact space and $M \subset X$ is a nonempty closed convex set, then the set of barycenters of the Borel probability measures with support $M$ coincides with the relative interior of $M$.

Proof. We note that in finite-dimensional spaces any probability Borel measure is Radon. It is also well-known (see [3]) that in such spaces the relative interior and the relative algebraic interior of $M$ coincide and are non-empty.

Now, let $a \in \operatorname{relint}(M)=\operatorname{core}(M)$ be any point. By the definition of the relative algebraic interior, for every $y \in M \subset$ aff $(M)$, the segment $[y, a]$ can be prolonged beyond the point $a$ within $M$. This means that $y \in V_{a}$, and thus $M \subset V_{a}$. Hence, by Theorem 1 (see also Remark 2), there exists $\mu \in \mathcal{P}(X)$ with supp $\mu=M$ and with barycenter $a$.

It remains to prove that if for some $a \in M$ one has $\overline{V_{a}}=M$, then $a \in$ relint $(M)$. Notice that $V_{a}$ is a non-empty convex set. Since we are dealing with a finite-dimensional space, $V_{a}$ has a non-empty relative interior, and $\operatorname{relint}\left(V_{a}\right)=\operatorname{relint}\left(\overline{V_{a}}\right)=\operatorname{relint}(M)$. Let $x \in \operatorname{relint}\left(V_{a}\right) \subset V_{a}$. It follows from the definition of $V_{a}$ that there exists a segment $[x, y] \subset M$ such that $a \in(x, y)$. Since $x$ also belongs to relint $(M)$, there exists an open neighborhood $U(x)$ of $x$ such that $U(x) \cap \operatorname{aff}(M) \subset M$.

By convexity of $M$, one obtains

$$
(1-\lambda)(U(x) \cap \operatorname{aff}(M))+\lambda y \subset M, \quad \lambda \in[0,1] .
$$

It is also easy to verify directly that

$$
(1-\lambda)(U(x) \cap \operatorname{aff}(M))+\lambda y=((1-\lambda) U(x)+\lambda y) \cap \operatorname{aff}(M), \quad \lambda \in[0,1] .
$$

Combining (15) and 16, and noticing that for $\lambda \in[0,1)$ the set $(1-\lambda) U(x)+$ $\lambda y$ is an open neighborhood of $(1-\lambda) x+\lambda y$, one sees that any point of $(x, y)$ belongs to relint $(M)$ by the very definition $(13)$ of the relative interior. In particular, this means that $a \in \operatorname{relint}(M)$.

2. It is tempting to think that Corollary 1.1 holds in infinite-dimensional spaces, too. Unfortunately, this is not the case even for Hilbert spaces, as the following counterexample shows. 
Let $X$ be the Hilbert space of real sequences endowed with the $l^{2}$-scalar product, and let $M$ be the Hilbert cube, a compact convex set,

$$
M=\prod_{k=1}^{\infty}\left[-\frac{1}{k}, \frac{1}{k}\right] \text {. }
$$

We take $a=\left\{a_{k}\right\}_{k=1}^{\infty} \in M$, where $a_{k}=\frac{1}{k+1}$. It is easy to construct a measure $\mu_{k} \in \mathcal{P}(\mathbb{R})$ with $\operatorname{supp} \mu_{k}=[-1 / k, 1 / k]$ such that

$$
\frac{1}{k+1}=\int_{[-1 / k, 1 / k]} x \mu_{k}(d x) .
$$

Having done that, consider the product of these measures restricted to $X$,

$$
\mu=\left.\bigotimes_{k=1}^{\infty} \mu_{k}\right|_{X}
$$

One usually defines the product of measures on the product of spaces, having in mind the product topology. Even though the corresponding induced topology on $X$ is strictly coarser than the $l_{2}$-norm topology, they both generate the same Borel sigma-algebra on $X$. Thus, it is clear that $\mu$ can be seen as a Borel measure on the Hilbert space $X$. Moreover, since $X$ is a complete and separable metric space, $\mu$ is Radon.

It is clear by construction that supp $\mu \subset M$. We prove the other inclusion by reductio ad absurdum.

Let $b \in M$, and suppose that $\mu\left(U_{\varepsilon}(b)\right)=0$ for some $\varepsilon>0$, where $U_{\varepsilon}(b)$ is the ball of radius $\varepsilon$ centered at $b$. Choose $N$ such that

$$
\sum_{n>N} \frac{4}{n^{2}}<\frac{\varepsilon^{2}}{2} \text {. }
$$

Then

$$
\begin{aligned}
0 & =\mu\left\{x \in X \mid \sum_{n=1}^{\infty}\left(x_{n}-b_{n}\right)^{2}<\varepsilon^{2}\right\} \geq \mu\left\{x \in M \mid \sum_{n=1}^{N}\left(x_{n}-b_{n}\right)^{2}<\varepsilon^{2} / 2\right\} \\
& =\bigotimes_{k=1}^{N} \mu_{k}\left\{x \in M \mid \sum_{n=1}^{N}\left(x_{n}-b_{n}\right)^{2}<\varepsilon^{2} / 2\right\} .
\end{aligned}
$$

The latter is positive, which gives a contradiction and yields supp $\mu=M$.

Now, we prove that $a$ is the barycenter of $\mu$. Thanks to the Riesz representation theorem, there exists $\left\{\lambda_{k}\right\}_{k=1}^{\infty} \in X$ such that for every $x=$ $\left\{x_{k}\right\}_{k=1}^{\infty} \in X$ one has

$$
\Lambda x=\sum_{k=1}^{\infty} \lambda_{k} x_{k} .
$$


By the definition of the barycenter we write

$$
\int_{X} \Lambda x \mu(d x)=\int_{M} \sum_{k=1}^{\infty} \lambda_{k} x_{k} \mu(d x)=\sum_{k=1}^{\infty} \lambda_{k} \int_{M} x_{k} \mu(d x)=\sum_{k=1}^{\infty} \lambda_{k} a_{k}=\Lambda a,
$$

where one can interchange the sum and the integral by dominated convergence since $M$ is a bounded set in $X$. This shows that $a$ is indeed the barycenter of $\mu$.

Next, we recall that in infinite-dimensional spaces the relative interior and relative algebraic interior do not necessarily coincide (see [3]). However, from (13) and (14) one sees that the former is a subset of the latter. Thus, it is sufficient to show that $a$ does not belong to the relative algebraic interior of $M$. We prove this again by contradiction.

Suppose that $a \in \operatorname{core}(M)$. Then the segment $[0, a]$ can be prolonged beyond $a$ within $M$. In other words, there exists $\alpha>0$ such that $(1+\alpha) a \in M$. The latter is equivalent to

$$
-1 / k \leq(1+\alpha) a_{k} \leq 1 / k, \quad k=1,2, \ldots
$$

Multiplying by $k+1$ and letting $k \rightarrow \infty$ yield

$$
-1 \leq 1+\alpha \leq 1,
$$

which contradicts $\alpha>0$ and concludes the proof.

3. Now, we describe the set of barycenters of measures on the space of probability measures. Let $K$ be a metric compact space and $X=\mathcal{M}(K)$ the space of signed finite Radon measures on $K$. By the Riesz-Markov theorem, $X$ can be identified with the topological dual $C^{*}(K)$ of the space $C(K)$ of continuous functions on $K$. We endow $C^{*}(K)$ with the weak-* topology $\sigma\left(C^{*}(K), C(K)\right)$. Having in mind the canonical embedding $C(K) \hookrightarrow$ $C^{* *}(K)$, one can say that this topology is the weakest topology which makes continuous all the functionals from $C^{* *}(K)$ that correspond to elements of $C(K)$. This topology is locally convex, as is the corresponding topology $\tau_{w}$ on $X$. The restriction of $\tau_{w}$ to the convex set $M=\mathcal{P}(K) \subset X$ of probability measures on $K$ produces the usual topology of weak convergence on $M$ and thus makes this set compact.

The barycenter $\mu \in X$ of a measure $\eta \in \mathcal{P}(X)$ is, by definition,

$$
\mu=\int_{X} \nu \eta(d \nu)
$$

if the latter integral exists in the weak sense. That is, since $\left(C^{*}(K)\right)^{\prime}=C(K)$, where $(\cdot)^{\prime}$ is the topological dual in the weak-* topology, $\mu$ is the barycenter of $\eta$ if and only if for every $f \in C(K)$,

$$
\int_{K} f(x) \mu(d x)=\int_{X}\left(\int_{K} f(x) \nu(d x)\right) \eta(d \nu) .
$$


Also, note that

$$
\mu=\int_{\operatorname{supp} \eta} \nu \eta(d \nu)
$$

and, by the Hahn-Banach separation theorem, one has $\mu \in \overline{\operatorname{co}(\operatorname{supp} \eta)}$.

The following result characterizes measures from $X$ with support $M$.

THEOREM 2. The set of barycenters of the measures from $\mathcal{P}(X)$ with support $M$ coincides with the set of the measures from $M$ with support $K$.

Proof. (a) First, we prove that the barycenter of a measure from $\mathcal{P}(X)$ with support $M$ is a measure from $M$ with support $K$.

Take any $\eta \in \mathcal{P}(X)$ such that $\operatorname{supp} \eta=M$, and let $\mu \in M$ be its barycenter. We prove that $\operatorname{supp} \mu$ is exactly $K$ by contradiction.

Indeed, suppose this is not the case. Then there exists a non-zero nonnegative continuous bounded function $f \in C_{b}(K)$ such that

$$
\int_{K} f(x) \mu(d x)=0 .
$$

Using (25) one gets

$$
\int_{M} \int_{K} f(x) \nu(d x) \eta(d \nu)=0,
$$

and since the integrand is non-negative,

$$
\int_{K} f(x) \nu(d x)=0,
$$

$\eta$-almost surely on $M$.

The latter, in fact, holds for all $\nu \in M=\mathcal{P}(K)$, due to continuity in $\nu$ of the left-hand side of 29 with respect to the topology of weak convergence.

Consequently, by choosing $\nu$ to be the delta measure at an arbitrary point of $K$, one immediately obtains

$$
f(x)=0, \quad x \in K,
$$

which contradicts $f \neq 0$ and concludes the proof of the claim.

(b) Now, assume that $\mu \in M$ and $\operatorname{supp} \mu=K$. Let

$$
A=\left\{\left(a_{1}, a_{2}, \ldots\right) \in[0,1]^{\infty} \mid a_{j} \geq 0, \sum_{j=1}^{\infty} a_{j}=1\right\}
$$

be a closed subset of $[0,1]^{\infty}$ endowed with the $l_{1}$-norm. Since $A$ is separable, there exists a Radon probability measure $\lambda$ on $[0,1]^{\infty}$ with support $A$ (see, e.g., the proof of Theorem 1). 
Let us also introduce the Radon probability measure $\lambda \otimes \mu^{\infty}=\lambda \otimes$ $\bigotimes_{j=1}^{\infty} \mu_{j}$ on $A \times K^{\infty}=A \times \prod_{j=1}^{\infty} K_{j}$, where the $\mu_{j}$ are copies of $\mu$, and the $K_{j}$ are copies of $K$. It is easy to see that

$$
\operatorname{supp}\left(\lambda \otimes \mu^{\infty}\right)=A \times K^{\infty} .
$$

Indeed, for any open neighborhood $U(c)$ of $c=\left(c_{a} ; c_{1}, \ldots\right) \in A \times K^{\infty}$, by the definition of the product topology, there exists an open set of the form

$$
U_{a}\left(c_{a}\right) \times \prod_{j=1}^{\infty} U_{j}\left(c_{j}\right),
$$

where $U_{a}\left(c_{a}\right) \subset A$ and $U_{j}\left(c_{j}\right) \subset K_{j}$ are open neighborhoods of $c_{a}$ and $c_{j}$, respectively, such that $U_{j}\left(c_{j}\right) \neq K_{j}$ only for finitely many $j \in \mathbb{N}$. Then, for large enough $N$ one has

$$
\left(\lambda \otimes \mu^{\infty}\right)(U(c)) \geq \lambda\left(U_{a}\left(c_{a}\right)\right) \prod_{j=1}^{N} \mu\left(U_{j}\left(c_{j}\right)\right)>0,
$$

which proves 32 .

The next step is to define the map $F: A \times K^{\infty} \rightarrow M$ by

$$
F(a, x)=\sum_{j=1}^{\infty} a_{j} \delta_{x_{j}} .
$$

It is easy to show that $F$ is continuous. Indeed, let $a^{(n)} \rightarrow a^{*} \in A$ in $l_{1}$-norm, and $x^{(n)} \rightarrow x^{*} \in K^{\infty}$ in the product topology. We will prove that $F\left(a^{(n)}, x^{(n)}\right)$ converges to $F\left(a^{*}, x^{*}\right)$ weakly. For every $f \in C(K)$,

$$
\begin{aligned}
\mid \int_{K} f(y) & F\left(a^{(n)}, x^{(n)}\right)(d y)-\int_{K} f(y) F\left(a^{*}, x^{*}\right)(d y) \mid \\
& \leq \sum_{j=1}^{\infty}\left|a_{j}^{(n)} f\left(x_{j}^{(n)}\right)-a_{j}^{*} f\left(x_{j}^{*}\right)\right| \\
& \leq \sup _{x \in K}|f(x)|\left\|a^{(n)}-a^{*}\right\|_{l_{1}}+\sum_{j=1}^{\infty} a_{j}^{*}\left|f\left(x_{j}^{(n)}\right)-f\left(x_{j}^{*}\right)\right| \rightarrow 0,
\end{aligned}
$$

where the latter term tends to zero thanks to the dominated convergence theorem. This proves the continuity of $F$.

Now, let us define the measure $\eta$ to be the pushforward of $\lambda \otimes \mu^{\infty}$ under $F$ given by

$$
\eta=\left(\lambda \otimes \mu^{\infty}\right) \circ F^{-1},
$$

which is readily verified to be a Radon probability measure. 
We prove that this measure is supported on $M$. Indeed, since it is known (see, e.g., [1, Ex. 8.1.6]) that

$$
\overline{F\left(A \times K^{\infty}\right)}=M,
$$

for every open neighborhood $U(\nu)$ of $\nu \in M$ there exists $(a, x) \in A \times K^{\infty}$ such that $F(a, x) \in U(\nu)$. Consequently, due to $F$ being continuous and due to 32 , one has $\eta(U(\nu))>0$, and thus, since $\nu$ is arbitrary, $\operatorname{supp} \eta=M$.

It remains to check that the barycenter of $\eta$ is $\mu$. One can write

$$
\begin{aligned}
\int_{M K} \int_{K} f(y) \nu(d y) \eta(d \nu) & =\int_{A \times K^{\infty} K} \int_{K} f(y) F(a, x)(d y)\left(\lambda \otimes \mu^{\infty}\right)(d a, d x) \\
& =\sum_{j=1}^{\infty} \int_{A} a_{j} \lambda(d a) \int_{K^{\infty}} f\left(x_{j}\right) \mu^{\infty}(d x) \\
& =\sum_{j=1}^{\infty} \int_{A} a_{j} \lambda(d a) \int_{K} f(x) \mu(d x)=\int_{K} f(x) \mu(d x),
\end{aligned}
$$

where we use the definition (36) of $\eta$, Fubini's theorem, and the dominated convergence to interchange the sum and the integrals.

According to (25), the formula (38) means exactly that the barycenter of $\eta$ is $\mu$. This concludes the proof of the theorem.

As a final remark we point out that our proof relies heavily on the fact that $K$ is compact. However, barycenters are well-defined for a wider class of Radon probability measures (with finite first moments). An open question of interest is to characterize such measures as well.

Acknowledgements. We would like to thank V. Bogachev for bringing the problem considered in this note to our attention, and A. Borichev for helpful discussions and valuable comments. Also, we greatly appreciate the detailed responses of the reviewers. Their remarks and comments, without a doubt, helped us improve our paper.

S.B. is supported by the European Research Council (ERC) under the European Union's Horizon 2020 research and innovation programme, grant 647133 (ICHAOS). A.M. is supported by the RFBR grants 14-01-90406, 14-01-00237 and the SFB 701 at Bielefeld University.

\section{References}

[1] V. I. Bogachev, Measure Theory, Vol. 2, Springer, Berlin, 2007.

[2] W. Rudin, Functional Analysis, McGraw-Hill, New York, 1991.

[3] C. Zălinescu, Convex Analysis in General Vector Spaces, World Sci., River Edge, NJ, 2002. 
Sergey Berezin

Aix-Marseille Université

Centrale Marseille, CNRS

Institut de Mathématiques de Marseille, UMR7373

39, Rue F. Joliot Curie

13453 Marseille, France

and

St. Petersburg Department of

V. A. Steklov Mathematical Institute of RAS

27, Fontanka

191023 St. Petersburg, Russia

ORCID: 0000-0003-4885-7794

E-mail: servberezin@yandex.ru

sergey.berezin@univ-amu.fr
Azat Miftakhov

Faculty of Mechanics and Mathematics Moscow State University

1, Leninskiye Gory 119991 Moscow, Russia

E-mail: miftakhov-af@tutanota.com 